\title{
Optimization of learning algorithms in multilayer perceptron for sheet resistance of reduced graphene oxide thin-film
}

\author{
Noor Aiman bin Aminuddin ${ }^{1}$, Nurlaila Ismail ${ }^{2}$, Marianah Masrie ${ }^{3}$, Siti Aishah Mohamad Badaruddin ${ }^{4}$ \\ ${ }^{1-3}$ College of Engineering, Universiti Teknologi MARA (UiTM), Malaysia \\ ${ }^{4}$ MIMOS Berhad, Technology Park Malaysia, Kuala Lumpur, Malaysia
}

\begin{tabular}{l}
\hline \hline Article Info \\
\hline Article history: \\
Received May 20, 2021 \\
Revised Jul 5, 2021 \\
Accepted Jul 14, 2021
\end{tabular}

\section{Keywords:}

Image classification

LM

MLP

Reduced graphene oxide

RP

SCG

Sheet resistance

\begin{abstract}
Multilayer perceptron (MLP) optimization is carried out to investigate the classifier's performance in discriminating the uniformity of reduced Graphene Oxide (rGO) thin-film sheet resistance. This study used three learning algorithms: resilient back propagation (RP), scaled conjugate gradient (SCG) and levenberg-marquardt (LM). The dataset used in this study is the sheet resistance of rGO thin films obtained from MIMOS Bhd. This work involved samples selection from a uniform and non-uniform rGO thin-film sheet resistance. The input and output data were undergoing data pre-processing: data normalization, data randomization, and data splitting. The data were divided into three groups; training, validation and testing with a ratio of $70 \%: 15 \%: 15 \%$, respectively. A varying number of hidden neurons optimized the learning algorithms in MLP from 1 to 10. Their behavior helped establish the best learning algorithms in discriminating MLP for rGO sheet resistance uniformity. The performances measured were the accuracy of training, validation and testing dataset, mean squared errors (MSE) and epochs. All the analytical work in this study was achieved automatically via MATLAB software version R2018a. It was found that the LM is dominant in the optimization of a learning algorithm in MLP for rGO sheet resistance. The MSE for LM is the most reduced amid SCG and RP.
\end{abstract}

This is an open access article under the CC BY-SA license.

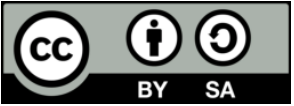

\section{Corresponding Author:}

Marianah Masrie

School of Electrical Engineering

College of Engineering

Universiti Teknologi MARA, 40450, Shah Alam, Selangor, Malaysia

Email: marianah@uitm.edu.my

\section{INTRODUCTION}

Graphene consists of one layer of carbon atoms organized in a very honeycomb pattern and may even be delineated as a one-atom-thick layer of graphite [1]-[4]. Graphene is reliable to be an electrical conductor for just one atom thick for remains light, flexible and transparent [5]-[7]. The most recognize technique has been developed to create large-scale continuous graphene films such as chemical vapour deposition (CVD), graphene epitaxial growth on silicon carbide ( $\mathrm{SiC}$ ) and graphene oxide reduction. Amongst these approaches, the oxide reduction proved to be a practical approach to produce graphene at a relatively low cost with optimal quality. Graphene Oxide (GO) is identified as an electrical insulator with low thermal conductivity due to the disruption of its sp2 bonding networks. To recover the hexagonal honeycomb lattice and electrical conductivity, the rGO must be produced in a high-temperature vacuum chamber with a certain degree of temperature. 
MLP is one of the preferred methods used for the classification and prediction of nanomaterials properties such as thin films, nanofluids, nanofiber, and nanocomposites reported in the previous research. Khosrojerdi et al. [8] predicted a thermal conductivity of graphene nanofluid using the multilayer perceptron (MLP) of an artificial neural network. Model accuracy was evaluated using square mean quadrature (RMS) indexes. The ANN algorithm was used to model $\mathrm{Cd}(\mathrm{II})$ elimination efficiency and optimize process variables of $\mathrm{Cd}(\mathrm{II})$ concentration, initial $\mathrm{pH}$ values, contact times and operating temperatures [9]. Amani et al. [10] performed multi-criteria modeling and optimization of the rheological and thermophysical properties of an environmentally-friendly covalently functionalized nanofluid containing graphene nanoplatelets (CGNPs). The Narx-ANN mathematical model was developed to shift the quartz resonator's frequency shift on GO langmuir bladgett thin-films [11]. The application of ANN to the classification and prediction of graphene nanomaterial is very minimal, but it is extensive for others [12]. Guo et al. [13] reported ten multilayer perceptrons were integrated into a random forest and multilayer perceptron (RF-MLP) model using the random forest (RF) method for predicting the dielectric loss of polyimide nanocomposite films. They also applied the MLP. A multilayer perceptron and a support vector machine (SVM) based on a PUK kernel were used to classify both the single-layer and three-layer polyimide nanocomposite films [14]. Konomi et al. [15] have developed a novel method to characterize thin-film conductivity in EFM based on feed-forward neural networks and evolutionary algorithms. MLP has also been conducted to predict the optical properties of Plasmonic thin-film solar cells and optimize their structures [16]. The MLP is also applied to predict the efficiency of a double-walled reactor using nanofluids as heat transfer and in predicting the nanofluids relative viscosity [17], [18]. Hassan et al. [19] have developed a model based on the prediction of R-squared value that can be implemented to estimate the values of specific heat capacity for nanofluids samples. For nanofiber materials, the MLP-based ANN model was used to predict the mean diameter of the electrospun fiber [20], [21]. Apart from this, two ANN models have been developed to model the elimination efficiency of nanomaterials heavy metals and the estimation of chemical material adsorption on nanocomposite [22], [23]. According to previous research, there is not yet report on the use of intelligent computing and neural networks technology to classify nanomaterial thin-film sheet resistance uniformity. This study has given rise to new challenges in the field of nanomaterial sheet resistance. MLP can provide the best model behaviour for a uniform sheet resistance.

This study proposes three learning algorithms of multilayer perceptron (MLP) classifier: resilient backpropagation (RP), scale conjugate gradient (SCG) and levenberg-marquardt (LM) for process modeling and accomplishing optimal coating parameters for investigating the nanomaterial thin film property. The algorithms have been developed to optimize the uniformity of rGO thin-film sheet resistance. The rGO sheet resistance datasets were acquired from the previous researcher in MIMOS Berhad. The data were processed beforehand and the datasets were used in three phases: training, validation and testing. The process continues with the development of the MLP model through RP, LM and SCG. Then, all three models developed models were tested and accepted once each model met performance criteria. Finally, the results obtained have also been validated experimentally.

\section{RESEARCH METHOD}

The experimental setup for the optimization of learning algorithms is depicted in Figure 1. The process begins with a data collection from MIMOS Berhad. The method of producing rGO sheet resistance datasets was started with the spray of graphene oxide (GO) with 3x and 4x spray passes on silicon dioxide ( $\mathrm{SiO} 2$ ) wafer by using an atomizer system developed by MIMOS Berhad [24]. The process was repeated for five runs of the experiment. The GO samples were then reduced through a high temperature of the thermal reduction process to produce rGO samples [25]. The four-point machine measured electrical conductivity, which is the sheet resistance at 49 different coordinate points distributed radially from the center of the whole 8-inch $\mathrm{SiO} 2$ wafer shown in Figure 2 right after the reduction process. The figure also illustrates the distribution of the sheet resistance values for $4 \mathrm{x}$ spray passes.

The datasets were undergoing data pre-processing where $70 \%$ was used for training, $15 \%$ for validation and the remaining data, $15 \%$ was used for testing. The process continued with the multilayer perceptron (MLP) training using two different datasets trained separately. In this process, learning algorithms were varied, which includes training using RP, LM and SCG. The neurons in the hidden layer were varied using pattern recognition network (patternet) function in MATLAB R2018a, set with 1 to 10 hidden neurons. Then it was followed by the validation and testing of the trained network for each learning algorithm. To accept the developed MLP model, the following performance was measured by the criteria of the confusion matrix, accuracy, sensitivity, specificity and precision that appeared in neural network training (nntraintool). The model was accepted if the model passed. But if not, it endured the data processing precise to either three processes as shown in Figure 1. 
As shown in Figure 3, one can see the architecture of multilayer perceptron (MLP) with input, hidden and output layers. The process starts from the first layer taking in inputs and the last layer producing output. In the middle of the layer is a hidden layer. The input layer has 49 neurons, representing 49 points on rGO sheet resistance by reading the 4-point probe machine. After that hidden layer is optimized from 1 to 10 neurons and the output layer has one neuron is representing the uniformity of the sheet resistance. Each perceptron is connected to every perceptron on the next layer. So that the information is constantly "feedforward" from one layer to the next; hence this network is also called a forward feed network.

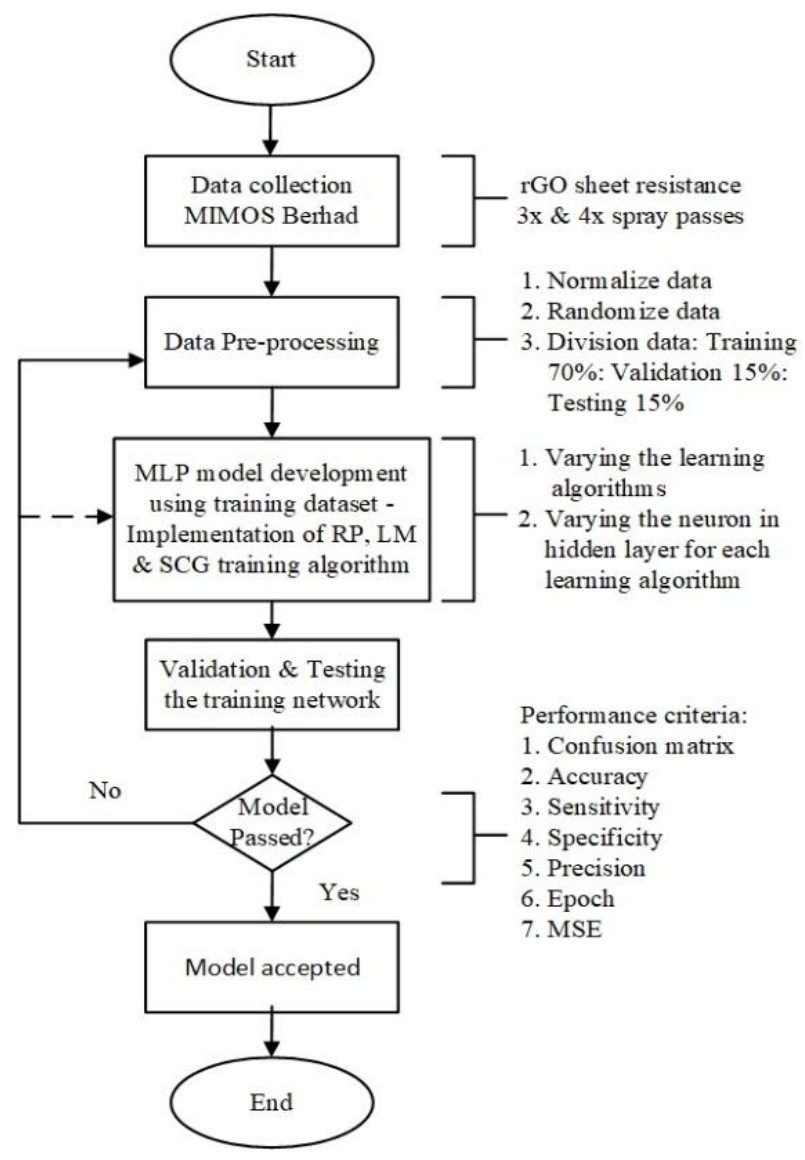

Figure 1. Flowchart of the experimental set-up in modeling for rGO sheet resistance classifier

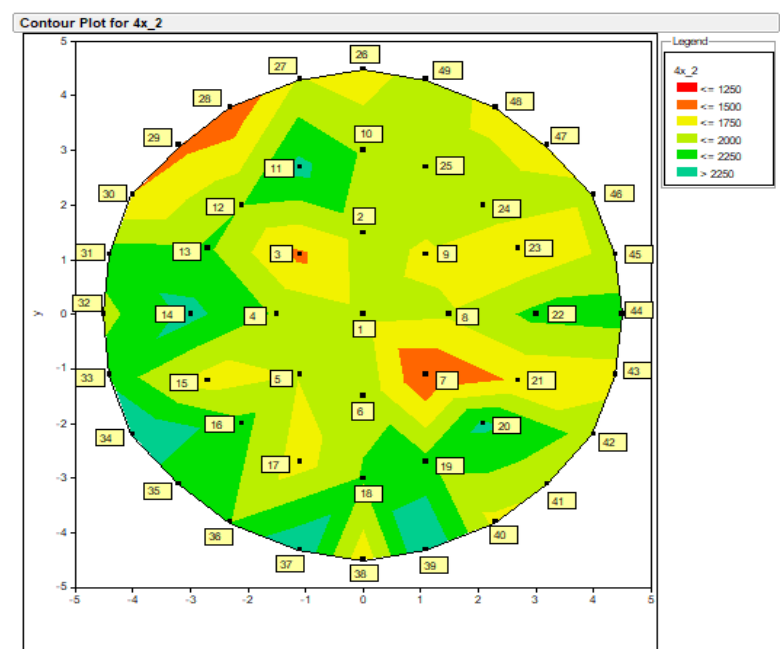

Figure 2. The contour plots for sheet resistance distribution on 49 points for $4 \mathrm{x}$ spray passes 


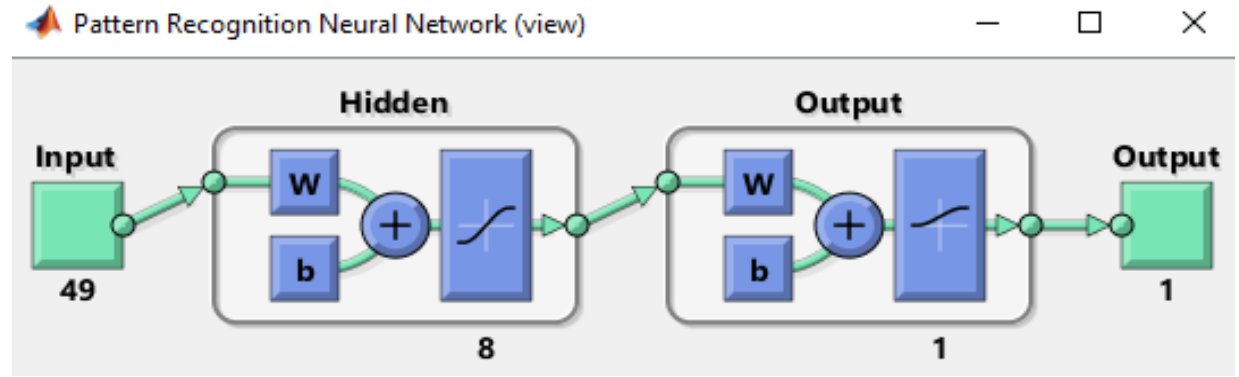

Figure 3. The MLP Architecture modeling for rGO sheet resistance classifier

\section{RESULTS AND DISCUSSION}

This section presents the classifier's performance in discriminating the uniformity of reduced graphene oxide (rGO) thin-film sheet resistance results obtained by optimizing RP, SCG and LM algorithms in testing, validation and training. Besides, these optimization learning algorithms, the number of epochs, the MSE value and the number of iterations are also highlighted.

Table 1 and Table 2 tabulate the accuracies for training, validation and testing for both datasets: $3 \mathrm{x}$ spray passes and 4x spray passes. The machine learning from the MATLAB software implementation optimized the model development that for all the training, validation and testing give the $100 \%$ accuracy for both datasets. From these data, it can be seen that the problem of uniformity of rGO sheet resistance is not too difficult for MATLAB version R2018a to optimize and the model is genuinely performing exceptionally well.

Table 1. Accuracy for training, validation and testing performance for RP, SCG and LM for 3x spray passes

\begin{tabular}{|c|c|c|c|}
\hline \multirow[t]{2}{*}{ No. of hidden neuron } & \multicolumn{3}{|c|}{ Accuracy $(\%)$} \\
\hline & Training & Validation & Testing \\
\hline 1 & 100 & 100 & 100 \\
\hline 2 & 100 & 100 & 100 \\
\hline 3 & 100 & 100 & 100 \\
\hline 4 & 100 & 100 & 100 \\
\hline 5 & 100 & 100 & 100 \\
\hline 6 & 100 & 100 & 100 \\
\hline 7 & 100 & 100 & 100 \\
\hline 8 & 100 & 100 & 100 \\
\hline 9 & 100 & 100 & 100 \\
\hline 10 & 100 & 100 & 100 \\
\hline
\end{tabular}

Table 2. Accuracy for training, validation and testing performance for RP, SCG and LM

\begin{tabular}{cccc}
\multicolumn{4}{c}{ for 4x spray passes } \\
\hline No. of hidden neuron & $\begin{array}{c}\text { Accuracy (\%) } \\
\text { Validation }\end{array}$ & Testing \\
\hline 1 & 100 & 100 & 100 \\
2 & 100 & 100 & 100 \\
3 & 100 & 100 & 100 \\
4 & 100 & 100 & 100 \\
5 & 100 & 100 & 100 \\
6 & 100 & 100 & 100 \\
7 & 100 & 100 & 100 \\
8 & 100 & 100 & 100 \\
9 & 100 & 100 & 100 \\
10 & 100 & 100 & 100 \\
\hline
\end{tabular}

Results illustrated in Table 3 and Table 4 show the average MSE versus the number of hidden neurons using RP, SCG and LM algorithms for 3x spray passes and 4x spray passes datasets. For 3x spray passes, the average MSE among the algorithms shows that LM was the best performance among the others. The smallest value for LM is only $7.26 \times 10^{-10}$ at hidden neurons 2 followed by SCG $\left(1.25 \times 10^{-7}\right)$ algorithm at hidden neurons10 and lastly, RP $\left(1.98 \times 10^{-7}\right)$ algorithm for nine hidden neurons. In $4 \mathrm{x}$ spray passes, the average MSE for LM is good compared to SCG and RP algorithms. The LM algorithm gives the minimum error, which is only $1.08 \times 10^{-9}$ at hidden neuron 7 while SCG $\left(1.20 \times 10^{-7}\right)$ at 8 hidden neurons and RP $\left(2.26 \times 10^{-7}\right)$ for 9 hidden neurons. Furthermore, SCG and RP algorithms still give the slightest error for both $3 \mathrm{x}$ spray passes and $4 \mathrm{x}$ spray passes, the range value close to 0 .

Table 5 and Table 6 summarize the parameter for the best-hidden neurons obtain in SCG, LM and RP algorithms (for both datasets: $3 x$ spray passes and $4 x$ spray passes. A similar finding was achieved by $3 x$ spray passes and $4 \mathrm{x}$ spray passes in which, for both sprays pass, the LM training algorithm outperforms others. It is shown by having the lowest MSE at $7.68 \times 10^{-10}$ ( $3 \mathrm{x}$ spray passes) and $1.08 \times 10^{-9}$ ( $4 \mathrm{x}$ spray passes). Besides that, the number of nodes in hidden neurons for LM is 2 (3x spray passes) and 7 (4x spray passes) accompanied with the epoch of 14 (3x spray passes) and 8 (4x spray passes). Interestingly, SCG and RP also give the smallest value of MSE, which is nearer to 0 and used the minimum of hidden neurons and epochs. 
Table 3. Average MSE versus the number of hidden neurons using RP, SCG and LM algorithms for 3x spray passes datasets

\begin{tabular}{cccc}
\hline No. of hidden neuron & $\begin{array}{c}\text { Mean square error (MSE) } \\
\text { RP }\end{array}$ & $\begin{array}{c}\text { Mean square error (MSE) } \\
\text { SCG }\end{array}$ & $\begin{array}{c}\text { Mean square error (MSE) } \\
\text { LM* }\end{array}$ \\
\hline 1 & $3.35 \times 10^{-6}$ & $3.21 \times 10^{-7}$ & $1.28 \times 10^{-8}$ \\
2 & $2.78 \times 10^{-6}$ & $3.10 \times 10^{-7}$ & $7.68 \times 10^{-10}$ \\
3 & $2.27 \times 10^{-6}$ & $1.47 \times 10^{-7}$ & $2.26 \times 10^{-8}$ \\
4 & $2.28 \times 10^{-6}$ & $1.28 \times 10^{-7}$ & $4.72 \times 10^{-9}$ \\
5 & $2.01 \times 10^{-6}$ & $1.84 \times 10^{-7}$ & $1.31 \times 10^{-8}$ \\
6 & $9.90 \times 10^{-7}$ & $2.19 \times 10^{-7}$ & $3.04 \times 10^{-9}$ \\
7 & $1.87 \times 10^{-6}$ & $1.33 \times 10^{-7}$ & $9.66 \times 10^{-9}$ \\
8 & $3.96 \times 10^{-7}$ & $1.70 \times 10^{-7}$ & $3.69 \times 10^{-9}$ \\
9 & $1.98 \times 10^{-7}$ & $1.56 \times 10^{-7}$ & $2.18 \times 10^{-9}$ \\
10 & $1.61 \times 10^{-6}$ & $1.25 \times 10^{-7}$ & $1.82 \times 10^{-9}$ \\
\hline
\end{tabular}

*The best performance

Table 4. Average MSE versus the number of hidden neurons using RP, SCG and LM algorithms for $4 \mathrm{x}$ spray passes datasets

\begin{tabular}{cccc}
\hline No. of hidden neuron & $\begin{array}{c}\text { Mean square error (MSE) } \\
\text { RP }\end{array}$ & $\begin{array}{c}\text { Mean square error (MSE) } \\
\text { SCG }\end{array}$ & $\begin{array}{c}\text { Mean square error (MSE) } \\
\text { LM* }\end{array}$ \\
\hline 1 & $4.17 \times 10^{-6}$ & $4.67 \times 10^{-7}$ & $2.37 \times 10^{-8}$ \\
2 & $2.39 \times 10^{-6}$ & $1.80 \times 10^{-7}$ & $2.41 \times 10^{-8}$ \\
3 & $9.30 \times 10^{-7}$ & $3.74 \times 10^{-7}$ & $8.72 \times 10^{-9}$ \\
4 & $1.96 \times 10^{-6}$ & $1.47 \times 10^{-7}$ & $5.87 \times 10^{-9}$ \\
5 & $1.28 \times 10^{-6}$ & $3.74 \times 10^{-7}$ & $6.74 \times 10^{-9}$ \\
6 & $2.13 \times 10^{-6}$ & $1.50 \times 10^{-7}$ & $2.43 \times 10^{-9}$ \\
7 & $2.26 \times 10^{-7}$ & $1.21 \times 10^{-7}$ & $1.08 \times 10^{-9}$ \\
8 & $1.37 \times 10^{-6}$ & $1.20 \times 10^{-7}$ & $5.20 \times 10^{-9}$ \\
9 & $3.85 \times 10^{-7}$ & $1.64 \times 10^{-7}$ & $3.40 \times 10^{-9}$ \\
10 & $1.48 \times 10^{-6}$ & $1.30 \times 10^{-7}$ & $3.87 \times 10^{-9}$ \\
\hline
\end{tabular}

Table 5. Parameters for the best RP, SCG and LM algorithms for 3x spray passes datasets

\begin{tabular}{lccc}
\hline \multicolumn{1}{c}{ Parameters } & RP & LM & SCG \\
\hline MSE & $1.98 \times 10^{-7}$ & $7.68 \times 10^{-10}$ & $1.25 \times 10^{-7}$ \\
Number of nodes in hidden neuron & 9 & 2 & 10 \\
Epochs & 5 & 14 & 22 \\
\hline
\end{tabular}

Table 6. Parameters for the best RP, SCG and LM algorithms for 4x spray passes datasets

\begin{tabular}{lccc}
\hline \multicolumn{1}{c}{ Parameters } & RP & LM & SCG \\
\hline MSE & $2.26 \times 10^{-7}$ & $1.08 \times 10^{-9}$ & $1.20 \times 10^{-7}$ \\
Number of nodes in hidden neuron & 7 & 7 & 8 \\
Epochs & 9 & 8 & 27 \\
\hline
\end{tabular}

The overall accuracy results for both $3 x$ and $4 x$ spray passes were quite similar. Therefore, only MLP final design parameter for 3x spray passes is discussed in this study. Table 7 summarizes the last design parameter for MLP architecture and the training parameter. The highest MSE found in LM by using dataset $3 \mathrm{x}$ spray passes which is $7.68 \times 10^{-10}$ with the epochs of 14 iterations. The number of the input layer is 49 points by reading from $\mathrm{rGO}$ sheet resistance and the hidden neurons of 2 neurons in the hidden layer obtained the lowest MSE. The output, which is the uniformity of rGO sheet resistance, is represented by 1 output layer. The confusion matrix, as depicted in Figure 4, shows that the accuracy of the training dataset is $100 \%$, and it is proven that LM outperforms others. This finding is supported by the results as tabulated in Table 8 in which the accuracy, sensitivity, specificity and precision are all $100 \%$.

Table 7. MLP design parameters

\begin{tabular}{lc}
\hline Parameter & Value \\
\hline Learning algorithms & LM \\
Dataset & $3 \times$ spray passes \\
Epochs & 14 \\
Mean Square Error (MSE) & $7.68 \times 10^{-10}$ \\
Number of Inputs layer & 49 \\
Number of nodes in the hidden layer & 2 \\
Number of the output layer & 1 \\
\hline
\end{tabular}

Indonesian J Elec Eng \& Comp Sci, Vol. 23, No. 2, August 2021: 686 - 693 


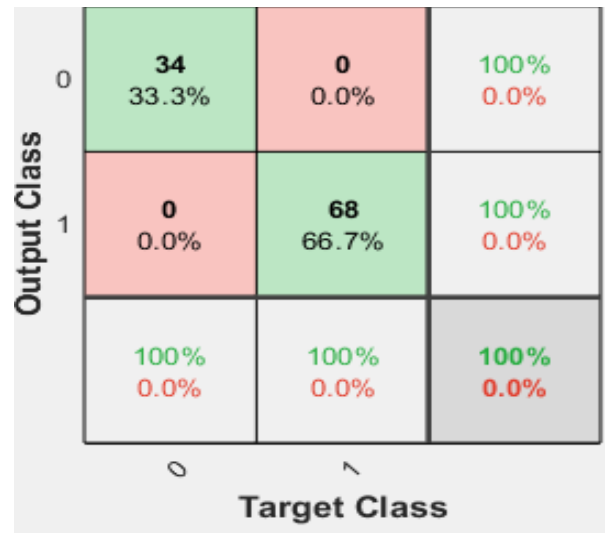

Figure 4. The MLP classification performance confusion matrix

Table 8. Accuracy, sensitivity, specificity and precision

\begin{tabular}{lc}
\hline Parameter & Value (\%) \\
\hline Accuracy & 100 \\
Sensitivity & 100 \\
Specificity & 100 \\
Precision & 100 \\
\hline
\end{tabular}

In general, it was found that levenberg marquardt (LM) training algorithms outperform others for all training, testing and validation datasets. This study proved that LM could obtain the lowest mean squared error (MSE) and performs 100\% accuracy for optimal behaviour (sensitivity, specificity and precision). Due to this, LM capable of approaching the direction of the steepest descent. This finding agrees that SCG is faster in computational time but more significant error compares to LM. This is because it avoids line search per learning iteration by the LM approach. Therefore, it is providing the lowest sum of squared errors.

\section{CONCLUSION}

The optimization algorithms in the MLP network for rGO thin-film sheet resistance uniformity for $3 \mathrm{x}$ and $4 \mathrm{x}$ spray passes were successfully performed in this study. It is clear that, amid three algorithms used in network training development to optimize rGO sheet resistance uniformity, LM gains the best. This study has been proven through uppermost accuracy (100\%) yielded by LM for testing, validation and training. However, the result is reduced to the MSE value only and the number of epochs and a number of the hidden neuron research area. This study is essential and beneficial, especially in the uniformity of graphene sheet resistance for classifier analysis.

\section{ACKNOWLEDGEMENTS}

The authors acknowledge funding from the Minister of Higher Education (MOHE) of Malaysia under the FRGS Grant No: 600-IRMI/FRGS 5/3/ (031/2019) and the School of Electrical Engineering, College of Engineering, Universiti Teknologi MARA (UiTM) for supporting this research.

\section{REFERENCES}

[1] A. Dahal, "Surface Science Studies of Graphene Interfaces," Graduate Theses, Department of Physics, University of South Florida, USA, 2015.

[2] Y. Han, "Electronic Properties of Graphene Tuned by Two-dimensional Crystals," Thesis, Department of Physics, Hong Kong University of Science and Technology, Hong Kong, 2015.

[3] V. K. Nagareddy, "Fabrication, functionalisation and characterisation of epitaxial graphene devices," Thesis, Department of Electrical and Electronic Engineering, Newcastle University, United Kingdom, 2015.

[4] M. Goosey, "A short introduction to graphene and its potential interconnect applications," Circuit World, vol. 38, no. 2, pp. 83-86, 2012, doi: 10.1108/03056121211222309.

[5] W. Wang, "Development of Nanocomposites Using Graphene Synthesized by Solvent Exfoliation Method," University of Toledo, 2014. 
[6] L. B. Modesto-López, M. Miettinen, T. Torvela, A. Lähde, and J. Jokiniemi, "Direct deposition of graphene nanomaterial films on polymer-coated glass by ultrasonic spraying," Thin Solid Films, vol. 578, pp. 45-52, 2015, doi: 10.1016/j.tsf.2015.01.073.

[7] D. Steinberg, R. Gerosa, F. Pellicer, S. Domingues, E. T. de Souza, and L. Saito, "Sub-300fs mode-locked erbium doped fiber laser using graphene oxide and reduced graphene oxide onto D-shaped optical fibers," in 2017 SBMO/IEEE MTT-S International Microwave and Optoelectronics Conference (IMOC), IEEE, 2017, pp. 1-3, doi: 10.1109/IMOC.2017.8121086.

[8] S. Khosrojerdi, M. Vakili, M. Yahyaei, and K. Kalhor, "Thermal conductivity modeling of graphene nanoplatelets/deionized water nanofluid by MLP neural network and theoretical modeling using experimental results," International Communications in Heat and Mass Transfer, vol. 74, pp. 11-17, 2016, doi: 10.1016/j.icheatmasstransfer.2016.03.010.

[9] M. Fan, et al., "Artificial neural network modeling and genetic algorithm optimization for cadmium removal from aqueous solutions by reduced graphene oxide-supported nanoscale zero-valent iron (nZVI/rGO) composites," Materials, vol. 10, no. 5, p. 544, 2017, doi: 10.3390/ma10050544.

[10] P. Amani and K. Vajravelu, "Intelligent modeling of rheological and thermophysical properties of green covalently functionalized graphene nanofluids containing nanoplatelets," International Journal of Heat and Mass Transfer, vol. 120, pp. 95-105, 2018, doi: 10.1016/j.ijheatmasstransfer.2017.12.025.

[11] K. Büyükkabasakal, S. C. Acikbas, A. Deniz, Y. Acikbas, R. Capan, and M. Erdogan, "Chemical sensor properties and mathematical modeling of graphene oxide langmuir-blodgett thin films," IEEE Sensors Journal, vol. 19, no. 20, pp. 9097-9104, 2019, doi: 10.1109/JSEN.2019.2926367.

[12] M. Kamran, S. Haider, T. Akram, S. Naqvi, and S. He, "Prediction of IV curves for a superconducting thin film using artificial neural networks," Superlattices and Microstructures, vol. 95, pp. 88-94, 2016, doi: 10.1016/j.spmi.2016.04.018.

[13] H. Guo, J. Zhao, and J. Yin, "Random forest and multilayer perceptron for predicting the dielectric loss of polyimide nanocomposite films," RSC advances, vol. 7, no. 49, pp. 30999-31008, June 2017, doi: 10.1039/C7RA04147K.

[14] H. Guo, J. Zhao, J. Yin, and L. Yao, "Structural testing of polyimide nanocomposite films with SAXS and SVMPUK," Polymer Testing, vol. 70, pp. 30-38, 2018, doi: 10.1016/j.polymertesting.2018.06.025.

[15] M. Konomi and G. Sacha, "Feedforward neural network methodology to characterize thin films by Electrostatic Force Microscopy," Ultramicroscopy, vol. 182, pp. 243-248, 2017, doi: 10.1016/j.ultramic.2017.07.015.

[16] M. Kaya and S. Hajimirza, "Surrogate based modeling and optimization of plasmonic thin film organic solar cells," International Journal of Heat and Mass Transfer, vol. 118, pp. 1128-1142, March 2018, doi: 10.1016/j.ijheatmasstransfer.2017.11.044.

[17] A. Mohammadi Ghahdarijani, F. Hormozi, and A. Haghighi Asl, "Convective heat transfer and pressure drop study on nanofluids in double-walled reactor by developing an optimal multilayer perceptron artificial neural network," International Communications in Heat and Mass Transfer, vol. 84, pp. 11-19, May 2017, doi: 10.1016/j.icheatmasstransfer.2017.03.014.

[18] H. Reza Ansari, M. Javad Zarei, S. Sabbaghi, and P. Keshavarz, "A new comprehensive model for relative viscosity of various nanofluids using feed-forward back-propagation MLP neural networks," International Communications in Heat and Mass Transfer, vol. 91, pp. 158-164, February 2018, doi: 10.1016/j.icheatmasstransfer.2017.12.012.

[19] M. A. Hassan and D. Banerjee, "A soft computing approach for estimating the specific heat capacity of molten saltbased nanofluids," Journal of Molecular Liquids, vol. 281, pp. 365-375, 2019, doi: 10.1016/j.molliq.2019.02.106.

[20] C. Yilmaz, D. Ustun, and A. Akdagli, "Usage of artificial neural network for estimating of the electrospun nanofiber diameter," in 2017 International Artificial Intelligence and Data Processing Symposium (IDAP), 2017, pp. 1-5, doi: 10.1109/IDAP.2017.8090329.

[21] C. Ieracitano, A. Paviglianiti, M. Campolo, A. Hussain, E. Pasero, and F. C. Morabito, "A novel automatic classification system based on hybrid unsupervised and supervised machine learning for electrospun nanofibers," IEEE/CAA Journal of Automatica Sinica, vol. 8, no. 1, pp. 64-76, 2021, doi: 10.1109/JAS.2020.1003387.

[22] A. H. Hamidian, S. Esfandeh, Y. Zhang, and M. Yang, "Simulation and optimization of nanomaterials application for heavy metal removal from aqueous solutions," Inorganic and Nano-Metal Chemistry, vol. 49, no. 7, pp. 217-230, 2019, doi: 10.1080/24701556.2019.1653321.

[23] M. Sadegh Mazloom, F. Rezaei, A. Hemmati-Sarapardeh, M. M. Husein, S. Zendehboudi, and A. Bemani, "Artificial intelligence based methods for asphaltenes adsorption by nanocomposites: Application of group method of data handling, least squares support vector machine, and artificial neural networks," Nanomaterials, vol. 10, no. 5, pp. 890, 2020, doi: 10.3390/nano10050890.

[24] M. Rofei Mat Hussin, S. Aishah Mohamad Badaruddin, N. Mohd Razali Mohd Nor, and M. Hilmy Azuan Hamzah, "Ultrasonic atomization of graphene derivatives for heat spreader thin film deposition on silicon substrate," Materials Today: Proceedings, vol. 7, 2019, pp. 763-769, doi: 10.1016/j.matpr.2018.12.072.

[25] M. Masrie, S. Badaruddin, M. Hussin, N. Nor, and J. Joe, "Rapid Reduction of Graphene Oxide Thin Films on Large-Area Silicon Substrate," in Journal of Physics: Conference Series, vol. 1535, no. 1, 2020, p. 012027, doi: 10.1088/1742-6596/1535/1/012027. 


\section{BIOGRAPHIES OF AUTHORS}
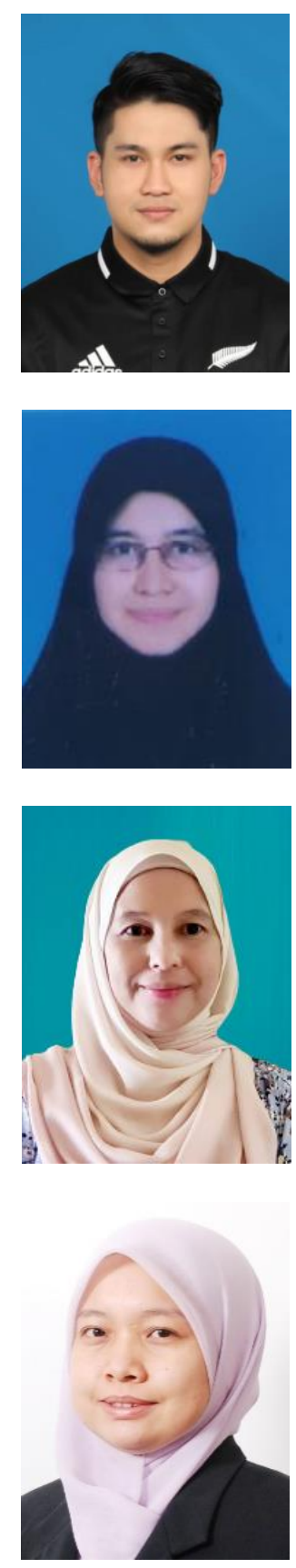

Siti Aishah Mohamad Badaruddin received the B.Sc. degrees in Electrical Engineering from University of Missouri Rolla, USA in 2000. She is currently a Senior Engineer in MIMOS's Advance Device Lab of Research \& Development department since 2017. Her major responsibility is heading the $2 \mathrm{D}$ nanomaterial group for the development of $2 \mathrm{DNM}$ mainly graphene and hexagonal boron nitride (hBN) in various application such as VOC, thermal management, and GaN process technology. She involved in the development of MIMOS atomizer system and its large area Graphene spray deposition process capability. Other main responsibility is producing IPs (patents, copyrights and trade secrets) and publications. Past experiences include 11 years as process engineers overseeing the process development, and optimization for thin film and wet process modules and 3 years as researcher for CMOS \& MEMS process group. 